\title{
Prevalencia de Anemia en niños de 6 a 24 meses de edad de tres Centros de Salud de la ciudad de La Paz
}

\author{
CINTHYA URQUIDI B. ${ }^{1}$, CLAUDIA VERA A. ${ }^{2}$, NOHEMI TRUJLLO B. ${ }^{3}$, HÉCTOR MEIIA S. ${ }^{4}$ \\ 1. Médico Epidemiólogo. \\ 2. Médico Cirujano. Centro de Iniciativa para el Desarrollo y Promoción en Salud. \\ 3. Médico Residente. Medicina Familiar. Hospital Obrero. La Paz. Bolivia. \\ 4. Pediatra Magister en Epidemiología Clínica. Hospital del Niño Dr. Ovidio Aliaga U. La Paz, Bolivia.
}

\begin{abstract}
Prevalence of anemia in children aged 6-24 months

Objetive: To determine the prevalence of anemia in children from three health centers of La Paz city. Design: Descriptive study. Methods: 114 children with ages between 6 to 24 months were enrolled. Capillary blood samples were obtained from a finger Prick with de use of aseptic standardized techniques; hemoglobin concentrations were measures immediately with a portable HemoCue. A cut off point adjusted for altitude was determinate for diagnosis $(13.6 \mathrm{~g} / \mathrm{dl})$. Results: The prevalence of anemia in all of the health centres was $86.6 \%$. In Bella Vista there was an $8 \%$ severe anemia, $10 \%$ Moderate anemia and $19 \%$ of mild anemia. In Chasquipampa $6 \%$ severe anemia, $11 \%$ and $12 \%$ moderate anemia and mild anemia respectively. In Villa Nuevo Potosí, 4\% severe anemia, 9\% moderate and 20\% mild anemia. The index of correlation of Spearman between the indicator tall for age and levels of hemoglobin was $0.2(\mathrm{p}=0.048)$. Conclusions: The high prevalence of anemia in children smaller than 2 years old, independently of gender, nutritional status and health center which they attend. Although the anemia has many causes, this high prevalence is suggestive that the diverse programs implemented for its control have not been effective.
\end{abstract}

(Key words: prevalence of anemia, anemia, iron deficiency).

Rev Soc Bol Ped 2006; 45 (3): 153-6

\section{RESUMEN}

Objetivo: Determinar la prevalencia de anemia en tres centros de salud. Diseño: Estudio descriptivo. Métodos: 114 niños de 6 a 24 meses de edad ingresaron al estudio, concurrentes a tres centros de salud de la ciudad de La Paz dependientes del Ministerio de Salud. Se midieron los niveles en sangre capilar con técnicas y personal estandarizado, muestras que fueron procesadas inmediatamente con el sistema fotómetro portable HemoCue. Para el diagnóstico de anemia se utilizó un punto de corte ajustado para la altura de 13,6 g/dl. Resultados: La prevalencia de anemia en los tres centros de Salud fue del 86,6\%. En el centro de Bella Vista se registró un $8 \%$ de anemia severa, $10 \%$ de anemia moderada y $19 \%$ de leve. En 
Chasquipampa, 6\% de anemia severa, 11\% y 12\% de anemia moderada y leve respectivamente. En Villa Nuevo Potosí, 4\% de severa, 9\% de moderada y 20\% de anemia leve. El índice de correlación de Spearman entre el indicador "talla para la edad" y niveles de hemoglobina fue de $0,2(p=0,048)$. Conclusiones: Existe una alta prevalencia de anemia en niños menores de 2 años, independientemente del género, estado nutricional o centro de salud al que asisten. Aunque la causa de anemia es multifactorial, la alta prevalencia es sugerente de que diversos programas implementados para su control, no han sido del todo efectivos. (Palabras clave: Prevalencia de anemia, anemia, deficiencia de hierro)

Rev Soc Bol Ped 2006; 45 (3): 153-6

\section{Introducción}

En 1985, De Maeyer y Adiels-Tegamn en su publicación "prevalencia de anemia en el mundo", la anemia nutricional es considerada como un importante componente de la anemia en general que afecta a más de 77 millones de niños y mujeres de América latina y el caribe; la deficiencia de hierro es considerada como la causa más común de este tipo de anemia, con el supuesto de que la prevalencia de anemia en adultos varones no es atribuida a la falta de hierro; los autores estimaron que más del $50 \%$ de la anemia en mujeres y niños es atribuible a la deficiencia de hierro ${ }^{1,2}$, aunque la causa de la anemia es multifactorial, es usualmente ferropénica, la que es comúnmente diagnosticada con bajas concentraciones de hemoglobina. La respuesta positiva de la hemoglobina a la suplementación con hierro, puede ser también usada como un parámetro de confirmación de anemia ferropénica ${ }^{3}$.

En 1993, en un consenso de la OMS y UNICEF, se determinó que la anemia sería considerada como un indicador de deficiencia de hierro, en vez de que la deficiencia de hierro pueda ser una causa contribuyente de anemia, ya que existen estadíos de leve a moderada deficiencia de hierro, donde la anemia está ausente y los tejidos ya están funcionalmente dañados ${ }^{3}$.

Se realizó una estimación cruda de una proporción de anemia atribuible a la deficiencia de hierro a través de una respuesta a la suplementación con hierro y folatos. Los resultados estiman que en niños escolares anémicos de la ciudad de La Paz, el 84\% es atribuible a carencia de hierro ${ }^{4}$.

Según la encuesta nacional de demografía y salud (ENDSA), realizada el año 2003, reporta que la prevalencia de anemia en niños de 6 a 59 meses es alta, pues casi uno de cada dos niños bolivianos padecen anemia (51\%). Al analizar la prevalencia de anemia según características demográficas, las diferencias más importantes se observan según la edad del niño, pues es muy alta entre los 10 y 11 meses, donde casi nueve de cada diez niños (89\%) padecen anemia.

La importancia en la disminución y control del problema, radica en la importancia del hierro en el desarrollo del sistema nervioso central y el cerebro; dado esto, numerosos estudios han demostrado que este tipo de anemia nutricional se encuentra estrechamente relacionada con una depresión tanto motora como mental en el desarrollo de los niños, la cual puede ser irreversible, por lo que su diagnóstico debe ser a temprana edad ${ }^{5}$.

\section{Material y Métodos}

Este estudio descriptivo que constituye la primera fase de un posterior estudio experimental, se llevó a cabo entre los meses de julio y diciembre del 2005, en tres centros de salud dependientes del Ministerio de Salud de la ciudad de La Paz: Centro Materno Infantil Bella Vista, Villa Nuevo Potosí y el Centro de Salud Chasquipampa, donde la población concurrente presenta un nivel socioeconómico homogéneo.

El tipo de muestreo fue no probabilístico de casos consecutivos, donde ingresaron al estudio todos los niños que consultaban al centro de salud ya sea por control, dental o consulta general.

Se tomaron muestras de sangre capilar con 
técnicas y personal estandarizados para la determinación de los niveles de hemoglobina, las muestras fueron procesadas con el Sistema Fotómetro B Hemoglobin HemoCue Portable. El punto de corte para el diagnóstico de anemia se ajustó según las recomendaciones del CDC para 3500 metros de altura, con lo que niños con valores de hemoglobina menores a 13,6 g/dl, fueron considerados como anémicos. Se recolectaron también algunas variables demográficas. No se incluyeron aquellos niños con evidencia clínica de desnutrición severa, enfermedad aguda grave que comprometa el estado general y aquellos provenientes de zonas de alto riesgo de Malaria o niños febriles.

De acuerdo a los percentiles 25,50 y 75 , se clasificó el grado de anemia como leve a valores de hemoglobina mayores de $12 \mathrm{~g} / \mathrm{dl}$ y menores a 13,6, moderada con valores de 10,5 a valores menores o iguales a $12 \mathrm{~g} / \mathrm{dl}$ y como severa con valores menores a $10,5 \mathrm{~g} / \mathrm{dl}$.

Este estudio constituye la primera fase de un ensayo clínico donde se determinó la adherencia a diferentes compuestos de hierro. Con un poder del $90 \%$ y un nivel de confianza del $95 \%$, se estimó una muestra total de 124 niños para los tres centros.

\section{Procesamiento estadístico}

Los datos previamente codificados fueron introducidos a una hoja de cálculo Excel (2003). Para el análisis, se utilizó el paquete estadístico para Windows SPSS 14.0. Se realizó previamente un análisis exploratorio de los datos. Las variables cualitativas, se expresaron con medias y sus respectivas desviaciones estándar, en el caso de existir anormalidad de la distribución se usó la media, como medida de tendencia central con sus respectivos rangos intercuartílicos. Las variables cualitativas se presentaron con frecuencias y proporciones.

\section{Resultados}

En total, se tamizaron 144 niños en los tres centros de salud, el $86,8 \%$ presentaban niveles de hemoglobina menores al punto de corte fijado, correspondientes a algún grado de anemia, de los cuales el $53 \%$ eran mujeres y $47 \%$ varones. Según los percentiles de hemoglobina, el $17,4 \%$ de los niños anémicos presentaban anemia severa, el 30,6\% anemia moderada y el $52,1 \%$ anemia leve.

Las prevalencia de anemia e índices antropométricos en cada centro de salud se muestran en la figura 1 y tablas 1,2 y 3 respectivamente.

\section{Centro materno infantil Bella Vista}

Se estudiaron 55 niños en este centro de salud, donde, $30(54,5 \%)$ correspondían al sexo masculino y $25(45,5 \%)$ al femenino. La edad mínima registrada fue de 6 y la máxima de 23 meses, la mediana de edad fue de 12 meses \pm 9 RI*. El peso mínimo registrado fue de 7,4 kilos y el máximo de 19 , con una mediana de $10 \pm 1,5$ RI. La mediana de talla fue de $74 \mathrm{~cm} \pm 7 \mathrm{RI}$,

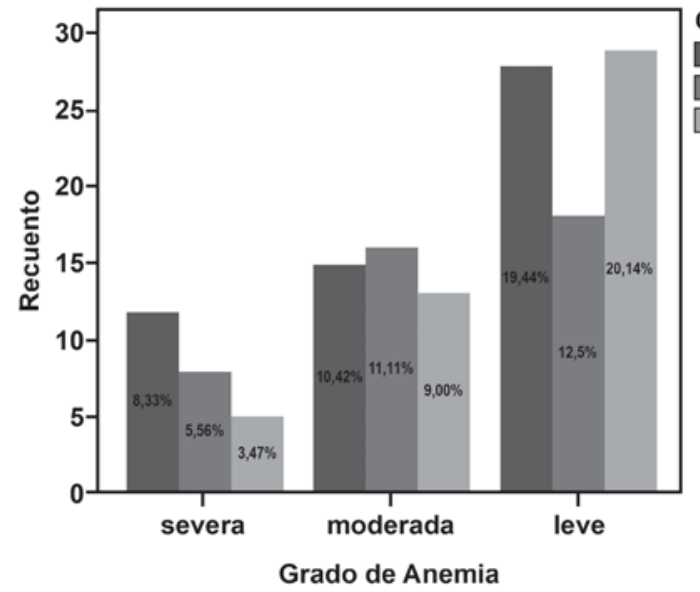

Volumen 79 - Número 3
Centro de Salud

$\square$ Bella Vista

Chasquipampa

$\square$ Villa Nuevo Potosí

Figura 1. Grado de anemia según Centro de Salud. 
Tabla 1. Estado nutricional según Indice Talla para la Edad

\begin{tabular}{lcccc}
\hline & $\begin{array}{c}\text { Bella Vista } \\
\mathbf{n = 4 8}\end{array}$ & $\begin{array}{c}\text { Chasquipampa } \\
\mathbf{n = 3 4}\end{array}$ & $\begin{array}{c}\text { Villa Nuevo Potosí } \\
\mathbf{n = 3 2}\end{array}$ & $\begin{array}{c}\text { Total } \\
\mathbf{n = 1 1 4}\end{array}$ \\
\hline Normal & 33 & 17 & 19 & $69(60,5)$ \\
$\begin{array}{l}\text { Desnutrición } \\
\text { Leve }\end{array}$ & 12 & 10 & & \\
Moderada & 2 & 5 & 9 & $31(27,2)$ \\
Grave & 1 & 2 & 3 & $10(8,8)$ \\
\hline
\end{tabular}

( ) expresado en \%; Correlación de Spearman: 0,2 ( $p=0,048)$

Tabla 2. Estado nutricional según Índice Peso para la Edad

\begin{tabular}{lcccc}
\hline & $\begin{array}{c}\text { Bella Vista } \\
\mathbf{n = 4 8}\end{array}$ & $\begin{array}{c}\text { Chasquipampa } \\
\mathbf{n = 3 4}\end{array}$ & $\begin{array}{c}\text { Villa Nuevo Potosí } \\
\mathbf{n = 3 2}\end{array}$ & $\begin{array}{c}\text { Total } \\
\mathbf{n = 1 1 4}\end{array}$ \\
\hline Normal & 33 & 17 & 19 & $69(60,5)$ \\
$\begin{array}{l}\text { Desnutrición } \\
\text { Leve }\end{array}$ & 9 & 11 & & \\
Moderada & 1 & 1 & 12 & $31(27,2)$ \\
Grave & 6 & 3 & 2 & 4 \\
\hline
\end{tabular}

( ) expresado en \%; Correlación de Spearman: 0,082 ( $p=0,471)$

Tabla 3. Estado nutricional según Índice Peso para la Talla

\begin{tabular}{lcccc}
\hline & $\begin{array}{c}\text { Bella Vista } \\
\mathbf{n = 4 8}\end{array}$ & $\begin{array}{c}\text { Chasquipampa } \\
\mathbf{n = 3 4}\end{array}$ & $\begin{array}{c}\text { Villa Nuevo Potosí } \\
\mathbf{n = 3 2}\end{array}$ & $\begin{array}{c}\text { Total } \\
\mathbf{n = 1 1 4}\end{array}$ \\
\hline $\begin{array}{l}\text { Normal } \\
\text { Desnutrición }\end{array}$ & 35 & 27 & 24 & $96(75,4)$ \\
Leve & 3 & 3 & 5 & $11(9,5)$ \\
Moderada & 0 & 2 & 0 & $2(1,8)$ \\
Grave & 9 & 2 & 2 & $13(11,4)$ \\
\hline ( ) expresado en \%; Correlación de Spearman: $0,072 \quad(p=0,377)$ &
\end{tabular}

con una mínima $66 \mathrm{~cm}$ y una máxima de $90 \mathrm{~cm}$ con respecto a la concentración de hemoglobina, el nivel mínimo registrado fue de $7 \mathrm{~g} / \mathrm{dl}$ y el máximo de 15,4 , con un valor promedio de 11,8 $\mathrm{g} / \mathrm{dl} \pm 1,6 \mathrm{DE} \dagger \dagger$ (IC 95\% 11,3-12,2).

Con respecto a la prevalencia de anemia, 48 de los 55 niños $(87,3 \%)$ presentaban niveles de hemoglobina menores al punto de corte. Según los niveles de hemoglobina, $12 / 55(21,8 \%)$ niños presentaban anemia severa, 15/55 (27,1\%) anemia moderada y $28 / 55(50,9 \%)$ leve.

\section{Centro de salud Chasquipampa}

De los 42 niños, 23 (54,8\%) eran mujeres y $49(45,2 \%)$ hombres. La mediana de edad fue de 12 meses \pm 11 RI. El peso mínimo registrado fue de 5,4 kilos y el máximo de 12,6 , con un promedio de 9,3 $\pm 1,5 \mathrm{DE}$ (IC 95\% 9,6, 10,4). La talla promedio fue de $73,4 \mathrm{~cm} \pm 5,7 \mathrm{DE}$ (IC $95 \% 71,6,75.2)$. El nivel mínimo de hemoglobina fue de $8,8 \mathrm{~g} / \mathrm{dl}$ y el máximo de 14,4 , con una media de $11,6 \mathrm{~g} / \mathrm{dl} \pm 1,6 \mathrm{DE}$ (IC 95\% 11,3, 12,2).

El 88\% eran anémicos (37/42). Según los niveles de hemoglobina, 8/42 (19\%) niños presentaban anemia severa, 16/55 (38\%) anemia moderada y 18/55 (43\%) leve.

\section{Centro Materno Infantil Villa Nuevo Potosí}

En este centro, 29/47 eran niñas (62\%) y el resto varones. La mediana de edad fue de 12 meses \pm 10 RI. El peso promedio de $10 \mathrm{~kg} \pm 1,6$ 
DE, con un mínimo de 6,25 y máximo de $12 \mathrm{~kg}$ (IC 95\% 8,5, 9,3). La mediana de talla fue de $72 \mathrm{~cm} \pm 7,2$ RI. Con respecto a la concentración de hemoglobina, el nivel mínimo registrado fue de $6,9 \mathrm{~g} / \mathrm{dl}$ y el máximo de 14,7 , con mediana de $12,3 \mathrm{~g} / \mathrm{dl} \pm 1,8 \mathrm{RI}$.

La prevalencia de anemia en este centro fue del 85\% (40/47). Según los niveles de hemoglobina, 5/47 (11\%) niños presentaban anemia severa, 13/47 (28\%) anemia moderada y 21/47 (62\%) leve.

\section{Discusión}

De acuerdo a la encuesta nacional de demografía y salud del 2003, la prevalencia ponderada de anemia en la ciudad de La Paz en niños menores de 2 años es del 75\%. Los resultados de este estudio multicéntrico concuerdan con este dato, siendo inclusive mayor, la prevalencia de anemia total en los tres Centros de Salud fue casi del $87 \%$. Un dato verdaderamente alarmante, principalmente por el impacto que tenga esta sobre el estado de salud de los niños, ya sea a corto o lago plazo; a corto plazo se preveen problemas con el desarrollo mental, motor y del sistema inmunológico y a largo plazo presentarán estatura corta, desempeño escolar bajo e incluso actividad física deficiente $^{6-10}$. La prevalencia de anemia fue similar en los tres centros de salud, la población de jurisdicción de estos centros presenta un nivel socioeconómico homogéneo, por lo que, tanto sus niveles de hemoglobina y estado nutricional no diferirá en gran medida. El hecho que estos resultados no varíen entre centros, supone que el trabajo realizado en la reducción de la anemia y aumento de las cobertura de hierro en un centro, no son mejores que en el otro centro y que el problema radicaría probablemente, en barreras propias que presenta el método de suplementación implementado para mitigar el problema $^{10}$. La correlación positiva moderada del índice "talla para la edad" con los niveles de hemoglobina, apoya una vez más lo que se sabe de causalidad entre anemia y baja talla por retardo del crecimiento. Se ha demostrado también la asociación del índice "peso para la ta- lla", como un indicador de desnutrición aguda, con los niveles bajos de hemoglobina, pero el tamaño de la muestra de este estudio no permite insinuar dicha asociación.

En este estudio, se demuestra la alta prevalencia de anemia en niños menores de 2 años, independientemente del género estado nutricional y centro de salud dependiente del Ministerio de Salud al que asisten. Aunque la causa de anemia es multifactorial, esta alta prevalencia es sugerente que los diversos programas implementados para su control, no han sido del todo efectivos. Quedan por investigar, los factores que influyen en la persistencia de esta alta prevalencia.

\section{Referencias}

1.- Demaeyer E M, Adiels-Tegman M: The prevalence of anemia in the world. Rapp Trimest Stat Sanit Mond 1985; 38: 302-16.

2.- Stoltzfus R: Defining iron-deficiency anemia in public health terms: a time for reflection. J Nutr 2001: 5657.

3.- Who/unicef/unu, ed: Iron deficiency anemia, assessment, prevention and control: a guide for programme managers. Who/nhd/01.3. Geneva: WHO, 2001

4.- Van den broek, Letsky E A: Etiology of anemia in pregnancyn south Malawi. Am J Clin Nutr 2000; 72: $247 \mathrm{~s}-56 \mathrm{~s}$.

5.- Inacg/ilsi: Why iron is important and what to do about it: a new perspective. Washington, DC 2002

6.- Dirren H, Logman $M H$, Barclay $D V$, Freire $W B$ : Altitude correction for hemoglobin. Eur J Clin Nutr 1994; 48: 625-32.

7.- World health organisation (WHO): Iron deficiency anemia: Assessment, prevention and control: a guide for programme Managers. Geneva: report of the department of nutrition for health and development, 2001 .

8.- Menéndez C, Todd J, Alonso P L, et al: The effects of iron supplementation during pregnancy, given by traditional birth attendants, on the prevalence of anemia and malaria. Trans Roy Soc Trop Med Hyg 1994; 88: 590-3

9.- Freire $W B$ : La anemia por deficiencia de hierro, estrategias de OPS/OMS para combatirla. Salud Pública Méx 1998; 40:199-205.

10.- Calvo E B, Gnazzo N: Prevalence of iron deficiency in children aged 9-24 month from large urban area of argentina. Am J Clin Nutr 1990; 52: 534-40. 